\title{
Neuralgia del trigémino: radiofrecuencia ganglio de Gasser
}

\author{
J. Pérez-Cajaraville ${ }^{1}$, M. Aseguinolaza Pagola ${ }^{1}$, P. Molina Tresaco 2 , J. Arranz Duran ${ }^{3}$ y D. Abejon Gonzalez ${ }^{4}$ \\ ${ }^{1}$ Unidad de Dolor. Clinica Universidad de Navarra. Pamplona. ${ }^{2}$ Servicio de Anestesiología, \\ Reanimación y Terapia del Dolor. Complejo Hospitalario de Navarra. Pamplona. ${ }^{3}$ Unidad de Dolor. \\ Servicio de Anestesiología. Hospital Universitario Nuestra Señora de Candelaria. Santa Cruz de Tenerife. \\ ${ }^{4}$ Unidad de Dolor. Hospital Universitario Puerta de Hierro Majadahonda. Madrid
}

Pérez-Cajaraville J, Asenilogaza M, Molina Tresaco P, Arranz Duran J, Abejon Gonzalez D. Neuralgia del trigémino: radiofrecuencia ganglio de gasser. Rev Soc Esp Dolor 2013; 20(2): 89-100.

\section{RECUERDO HISTÓRICO}

La célebre frase "Hoy dueles, mañana serás solo un recuerdo..." parecía no estar relacionada con la neuralgia del trigémino (NT) hasta la aparición de nuevas técnicas invasivas.

El Papiro de Ebers (1550 a. C.) describe, con gran detalle, el empleo del opio para las cefaleas del dios Ra (1), pero es el médico griego Areteo de Capadocia (50-150 a. C.) el que realiza la primera descripción inequívoca de una migraña $(2,3)$, “dolor en el que el espasmo distorsiona el semblante...".

En 1667, John Locke describe y trata una afección facial dolorosa trigeminal con ácido sulfúrico sobre la cara de la Duquesa de Northumberland.

Nicolás André (1756) describe la entidad clínica como "tic doloroso" y considera el abordaje y la destrucción directa del nervio como tratamiento. No obstante es John Fothergill, el primero en describir completamente el cuadro en 1773, ante la Sociedad Médica de Londres.

En 1911, Hartel y Tapias realizan la primera alcoholización del ganglio de Gasser por vía percutánea (4), y en 1931, Kirschener sugiere la destrucción del ganglio por procedimientos no quirúrgicos, usando radiofrecuencia, aunque relamente el padre de la RF Gasser fue Sweet, mejorando resultados y reduciendo complicaciones.

\section{RECUERDO ANATÓMICO}

El nervio trigémino es el de mayor grosor entre los doce pares craneales, también llamado quinto par (V par). Su nombre (del latín: trigeminus, quiere decir "trillizos") deriva del hecho de poseer tres grandes ramas/nervios principales: oftálmica $\left(\mathrm{V}_{1}\right)$, maxilar $\left(\mathrm{V}_{2}\right)$ y mandibular $\left(\mathrm{V}_{3}\right)$.

Las ramas $\mathrm{V}_{1}$ y $\mathrm{V}_{2}$ son sensoriales mientras que la rama mandibular tiene componente sensitivo y motor.

Es un nervio mixto, pues tiene un núcleo motor y otro sensitivo, corresponde al primer arco visceral o branquial.

\section{Conexiones}

La raíz sensitiva es considerablemente mayor que la motora y está formada por prolongaciones periféricas (preganglionares) y prolongaciones centrales (postganglionares) de las neuronas unipolares situadas en el ganglio trigeminal (llamado de Gasser o ganglio semilunar).

\section{Recorrido periférico (Tabla I)}

- Recorrido del nervio oftálmico: poco después de su salida del ganglio de Gasser, se dirige hacia adelante, introduciéndose en la pared lateral del seno cavernoso, en donde se divide en tres ramas: frontal, nasociliar y lacrimal; las cuales penetran en la órbita por la hendidura esfenoidal. 
TABLA I. RAMAS DEL TRIGÉMINO

\begin{tabular}{lll}
\hline Rama oftálmica $(V 1)$ & Rama maxilar $(V 2)$ & Rama mandibular $(V 3)$ \\
\hline N. Frontal & N. dentario posterior & N. dentario \\
- N. supratroclear & N. dentario anterior & N. lingual \\
- N. supraorbitario & N. esfenopalatino & - N. submandibulares \\
N. nasociliar & N. palatino mayor & - N. submentonianos \\
- N. infratroclear & N. palatino menor & N. alveolar inferior \\
- N. etmoidal anterior & & - N. mentoniano \\
- N. ciliares & & - N. milohioideo \\
N. lacrimal & & N. auriculotemporal \\
& & N. bucal \\
\hline
\end{tabular}

- Recorrido del nervio maxilar: igualmente que el anterior, poco después de su salida del ganglio de Gasser, se sitúa en la pared lateral del seno cavernoso. Abandona el cráneo por el agujero redondo mayor y llega a la fosa pterigo-maxilar donde se divide en varias ramas. La rama más importante es el nervio infraorbitario que penetra en la base de la órbita por el agujero suborbitario, pasando por el canal suborbitario y alcanzando el maxilar superior en su rama ascendente, por delante de la carilla articular del malar. Al salir del agujero suborbitario da ramas que se distribuyen por la mejilla.

En su recorrido por el conducto orbitario da las ramas dentarias superiores y anteriores, que van a inervar los dientes de la parte más anterior del maxilar superior. Hay otra rama antes del recorrido intraóseo que es el nervio cigomático que va a anastomosarse con el nervio lacrimal.

El nervio maxilar tiene aún dos ramas más que son los nervios pterigopalatinos que confluyen en el ganglio esfenopalatino y a partir de aquí se forman los nervios palatinos, menor y mayor, que se distribuyen por la mucosa del paladar.

- Recorrido del nervio mandibular: abandona el cráneo por el agujero oval y llega a la cara externa de la apófisis pterigoides, o sea por la fosa cigomática, que está ocupada por los músculos pterigoideos interno y externo. El nervio transcurre entre ellos y da finalmente varias ramas: lingual, dentaria, alveolar inferior, auriculotemporal y bucal.

Además de todas estas ramas sensitivas, el nervio mandibular tiene también ramas exclusivamente motoras destinadas a inervar los músculos masticadores. Entre ellas: una rama para el temporal, una para el masetero, y otra que acompaña al nervio dentario inferior y va al vientre anterior del digástrico y músculo milohioideo. Estas ramas conducen la sensibili- dad a diferentes partes de la cabeza, e ingresan en el cráneo por diferentes agujeros que conviene tener en cuenta (Tabla II).

Las áreas de distribución cutánea (dermatomas) de las tres ramas son muy fiables y bien limitadas, al contrario que en el resto el cuerpo (Fig. 1).

\section{NEURALGIA DEL TRIGÉMINO}

\section{Introducción}

La neuralgia del trigémino (NT) puede definirse como aquel trastorno donde el síntoma dominante es el dolor facial, relacionándose con fibras periféricas o centrales del nervio trigémino. La NT es uno de los dolores más intensos conocidos, caracterizado por breves y lancinantes paroxismos de dolor facial que duran unos segundos, llegando a 1-2 minutos. Son sinónimos: tic douloureux, neuralgia trifacial, neuralgia trigeminal mayor, y neuralgia esencial de trigémino.

Esta enfermedad, conocida inicialmente (año 1756) como tic doloroso, fue definida por Trauseau como "neuralgia epileptiforme espasmódica" en 1885.

\section{Epidemiología}

La NT es la neuralgia más frecuente de los nervios craneales, afectando a 4-10 de cada 100.000 habitantes cada año $(5,6)$. Se produce en personas mayores de 50 años en un $80 \%$ de las ocasiones, con un pico de incidencia entre los 60-70 años (7).

El lado derecho suele afectarse algo más frecuentemente (60\%), siendo bilateral en solo 1-6\% de los casos. Es 1,8-4,3 veces más común en mujeres, según diferentes series (8). 
TABLA II. ANATOMÍA RAMAS TRIGEMINALES

\begin{tabular}{|c|c|c|c|}
\hline & Rama oftálmica (VI): & Rama maxilar (V2): & Rama mandibular (V3): \\
\hline \multirow[t]{8}{*}{ Inervación sensitiva } & Piel de la frente & Piel de región malar & Músculos masticadores \\
\hline & Nariz & Piel región maxilar & Labio inferior \\
\hline & Conjuntiva de los ojos & Mucosa nasal & 2/3 Anteriores de la lengua \\
\hline & Meninges & Labio superior & Mucosa bucal \\
\hline & Párpados & Dientes & Dientes \\
\hline & Mucosa seno paranasal & Paladar óseo & $\begin{array}{l}\text { Parte del oído y meato } \\
\text { auditivo externo }\end{array}$ \\
\hline & & $\begin{array}{l}\text { Mucosa seno maxilar } \\
\text { superior }\end{array}$ & $\begin{array}{l}\text { Maxilar inferior y } \\
\text { preauditiva }\end{array}$ \\
\hline & & & $\begin{array}{l}\text { Sensibilidad propioceptiva } \\
\text { de la ATM }\end{array}$ \\
\hline Ingresa al cráneo por & $\begin{array}{l}\text { Hendidura esfenoidal } \\
\text { (fisura orbitaria sup.) }\end{array}$ & $\begin{array}{l}\text { Agujero redondo mayor } \\
\text { (rotundo) }\end{array}$ & Agujero oval \\
\hline Responsable del reflejo & $\begin{array}{l}\text { Corneal, lacrimal y } \\
\text { parpadeo }\end{array}$ & Del estornudo & \\
\hline
\end{tabular}

Un 50\% de pacientes reflejan un "punto gatillo" o desencadenante de dolor. La rama que más frecuentemente está expuesta a la aparición del dolor es la tercera, la segunda en aproximadamente un $25 \%$, siendo $\mathrm{V}_{1}$ la menos afectada (2\%). En un $40-45 \%$ de las ocasiones están involucradas la $2 .^{a}$ y $3 .^{a}$ ramas juntas.

Se ha estimado en un 5\% el porcentaje de enfermos con historia familiar de NT. Estudios epidemiológicos han demostrado como factores de riesgo la esclerosis múltiple e hipertensión.

\section{Etiología}

Hoy en día aún no tenemos claro el porqué de la NT, el dolor parece ser originado por un fenómeno de desmielinización y agrupamientos axonales en algún tramo de su recorrido, por compresión o inflamacion. A pesar de que existe una pequeña proporción de neuralgias del trigémino que se asocian a determinadas enfermedades, un gran porcentaje de las mismas continúan siendo de carácter idiopático, sugiriéndose diversas teorías etiológicas:

- La más difundida es la "teoría vascular", dado que la mayoría de los pacientes presentan compresión de las raíces del trigémino por vasos tortuosos o aberrantes. Entre el 80 y 90\% de las NT llamadas idiopáticas se deben en realidad a la compresión del nervio, cerca de su salida del tronco encefálico, por una vena o arteria que formen un 'bucle'. Existe evidencia científica que demuestra que hasta en un $25 \%$ de la poblacion que

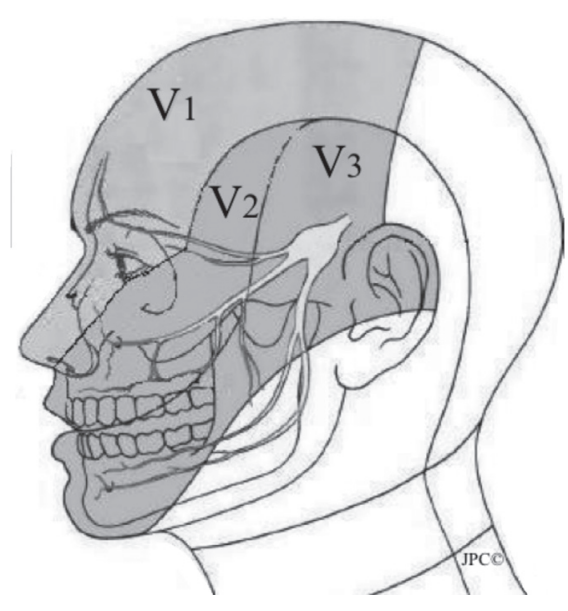

Fig. 1. Dermatografía nervio trigémino.

no padecen NT puede existir un contacto anatómico entre nervio y arteria sin clínica (9).

- La "epileptógena" postulada por Fromm y cols. (10). La irritación crónica de terminaciones nerviosas trigeminales podría inducir una alteración en sistemas inhibitorios segmentarios (a nivel de núcleos sensitivos del trigémino) y por tanto, un aumento en la actividad de estos núcleos debido a la aparición de potenciales de acción ectópicos. El incremento en la actividad de fibras aferentes primarias junto con el deterioro de los mecanismos inhibitorios, en los núcleos sensitivos del trigémino, llevarían a la producción de descargas paroxísticas de las interneuronas de dichos núcleos, 
TABLA III. DIAGNÓSTICO DIFERENCIAL NT

\begin{tabular}{lll}
\hline & Esencial & Sintomática \\
\hline Etiología & Desconocida & $\begin{array}{l}\text { Lesión, } \\
\text { compresión }\end{array}$ \\
Edad & $>50$ años & $<50$ años \\
Calidad dolor & Paroxismos & Continuo \\
Puntos gatillo & Frecuente & Infrecuente \\
Déficit neurológico & No & Frecuente \\
Respuesta a fármacos & $\begin{array}{l}\text { Buena, } \\
\text { inicialmente }\end{array}$ & Escasa \\
Afectación & Unilateral & Uni o bilateral \\
\hline
\end{tabular}

en respuesta a estímulos táctiles y, consecuentemente, a la provocación de las crisis dolorosas.

- Otras teorías que se han postulado son la irritación mecánica del ganglio de Gasser, la hipermielinización degenerativa o desmielinización segmentaria y síndromes talámicos parciales desencadenados por deficiencia de riego sanguíneo ocasionado por la edad.

- En un 2-3\% de las ocasiones coexiste esta neuralgia con la esclerosis múltiple, por lo cual ante toda NT deberíamos realizar un completo y extenso examen neurológico en busca de la esclerosis en placas (sospecharla ante todo paciente joven, con déficit sensorial de la cara, alteraciones de la visión, cambios en el fondo de ojo, etc.).

\section{Clínica y diagnóstico}

El diagnóstico de NT sigue siendo clínico, aunque consideramos necesarias pruebas complementarias como, radiografía dental por posibles problemas odontológicos y RM cerebral para descartar otras causas del dolor (tumoral, esclerosis múltiple, etc.) sobre todo en pacientes jóvenes con síntomas atípicos y los que no respondan a la terapia médica inicial (11).

El diagnóstico, en muchas ocasiones, se retrasa o queda enmascarado por dolencias faciales frecuentes, como un dolor dental o migrañas.

Tras una primera orientación diagnóstica de NT, el segundo paso ha de ser diferenciar neuralgia esencial y sintomática (Tabla III).

La NT se clasifica en:

- Primarias, esenciales o idiopáticas.

- Secundarias. Por compresión: neoplásica, traumática, las instrumentaciones bucales, vascular, infecciosa, entre otras.

- Lesiones difusas: esclerosis múltiple, neurosífilis y el herpes zóster.
TABLA IV. CARACTERÍSTICAS NT IDIOPÁTICA

\begin{tabular}{l}
\hline Síntomas clásicos: \\
- Paroxístico y fulgurante \\
- Provocable en zonas gatillo \\
- Sigue el territorio de distribución \\
- Unilateral \\
- No hay pérdida de sensibilidad \\
\hline Síntomas atípicos: \\
- Continuo \\
- Puede irradiarse a cuello u occipucio \\
- Hipoestesia o hiperalgesia espontánea \\
\hline
\end{tabular}

La NT esencial o primaria, con mucho la más frecuente, en la cual no se observan alteraciones neurológicas en la exploración clínica y no se objetiva con las técnicas diagnósticas habituales ninguna lesión orgánica responsable del cuadro (12). Puede presentarse bajo dos aspectos diferentes según la clínica:

1. Neuralgia idiopática típica del trigémino: el cuadro clínico es muy específico y nos permite el diagnóstico con bastante certeza. Las manifestaciones clínicas se encuentran resumidas en la tabla IV.

2. Neuralgia idiopática atípica del trigémino:

- Dolor urente o quemante.

- Dolor continuo, dura varios minutos, horas, incluso todo el día.

- Pueden existir ocasionalmente paroxismos sobreañadidos (superpuestas a este dolor pueden haber crisis de dolor agudo más intenso, en ocasiones asociadas a signos de hiperactividad autonómica). En algunos casos los pacientes refieren alteraciones sensitivas en el territorio afecto.

- Dolor, unilateral, está localizado en el territorio de una o más ramas del nervio.

- No puede provocarse el dolor con estímulos en las zonas gatillo, no desencadenándose ni con la masticación ni con el habla.

\section{Estudios complementarios}

Varias exploraciones complementarias sirven para evidenciar las posibles lesiones estructurales susceptibles de provocar síntomas de NT y confirmar sospecha de NT no esencial (15\% de los casos).

- Pruebas de imagen:

- Radiología: craneofacial, base del cráneo y columna cervical.

- RM: la exploración más eficaz en patología del trigémino. Su indicación de forma sistemática no está clara, aunque debe indicarse en pacientes con pérdida de sensibilidad, menores de 40 años, sinto- 
matología bilateral y NT refractarias a tratamiento conservador (13).

- Pruebas neurofisiológicas:

- EEG: pequeño porcentaje de tumores de ángulo pontocerebeloso muestran brotes de actividad delta focal, de proyección temporal en uno u otro hemisferio.

- EMG: en neuropatías o radiculopatías motoras del trigémino, puede demostrar denervación. La exploración neurofisiológica del reflejo de parpadeo es útil en el estudio del trigémino y sus conexiones trigémino-faciales a nivel pontino y bulbar.

Ningún estudio neurofisiológico es primordial para el diagnóstico de NT idiopática.

\section{Tratamiento}

Ante un paciente con NT la literatura médica parece estar totalmente de acuerdo en que la primera opción universalmente aceptada es la farmacológica y solo cuando los pacientes no obtienen alivio del dolor, debido a la recurrencia del mismo o por los efectos secundarios adversos de los medicamentos, serían candidatos a técnicas quirúrgicas mínimamente invasivas o cirugías mayores.

La terapia farmacológica suele ser efectiva inicialmente, pero a menudo, pierde eficacia al cabo del tiempo, requiriendo una solución invasiva $(25-50 \%$ de los pacientes diagnosticados de NT esencial).

\section{Intervencionista}

La tasa de mala respuesta a la medicación, como ya hemos mencionado, varía entre un $25-50 \%$ según diferentes series.

Las técnicas intervencionistas, aplicadas a NT idiopática, se podrían subdividir en tres grandes bloques dependiendo del nivel anatómico al que actuemos: periférico, lesiones a nivel del ganglio de Gasser o técnicas de cirugía abierta, (Tabla V). La NT secundaria, generalmente requiere de cirugía abierta.

\section{Bloqueos a nivel periférico}

Todos estos procedimientos tienen como objetivo lesionar el nervio que produce el dolor (punto trigger) (p. ej. nervio mentoniano, lingual, bucal, infraorbitario, etc.) mediante procedimientos químicos (inyección de alcohol (14) o glicerol), térmicos (radiofrecuencia -RF-, crioterapia) o físicos (neurectomía), con el fin de bloquear la conducción del estímulo doloroso. Todos fáciles de realizar, repetibles, bien tolerados y presentan una baja morbilidad con una mortalidad nula. Sin embargo, generalmente, confieren un alivio del dolor de corta duración. De todos ellos la radiofrecuencia pulsada (RFP) del nervio periférico parece tener un gran futuro terapéutico (Fig. 2).

La inyección de alcohol requiere una técnica precisa para depositarlo allí donde realmente se requiere, puesto que conlleva unos efectos secundarios locales de cierta importancia, en forma de edema y malestar que duran unos cuantos días (15). Esta técnica proporciona un mayor periodo de remisión del dolor que la inyección de glicerol y que la inyección de estreptomicina y lidocaína (16). Hasta la fecha se consideraba la crioterapia como la técnica que presenta menos complicaciones postoperatorias de todas las periféricas, en nuestra experiencia obtenemos mejores resultados con la radiofrecuencia pulsada, sin complicaciones.

Estos procedimientos son realmente eficaces si logramos identificar puntos desencadenantes de la NT.

Técnicas a nivel del ganglio de Gasser

- Rizotomía percutánea retrogasseriana mediante glicerol: consiste en la inyección de glicerol $(0,2-$ $0,5 \mathrm{ml})$, mediante aguja espinal 20G, tras identificación de la cisterna subaracnoidea de Gasser en el cavum de Meckel. El glicerol es un agente no neurolítico que produce un bloqueo prolongado del nervio y posiblemente provoque un retardo en la actividad excitatoria de las fibras nerviosas por interferencia en la resíntesis de proteínas. Sus principales desventajas son que el dolor puede tardar entre 7 y 19 días en disminuir, los fracasos iniciales son elevados y existe un alto grado de posibilidad de recidiva. La técnica del glicerol puede estar indicada en neuralgias de primera rama y neuralgias de trigémino bilaterales por esclerosis en placas (18). Tasa de recurrencia del dolor del $54 \%$ a los 4 años.

- Compresión-descompresión percutánea del ganglio de Gasser mediante sonda de Fogarty: descrita en 1978 por Mullan y Lichtor introduciendo por vía percutánea una sonda de Fogarty del n. ${ }^{\circ} 4$ a través de un trocar colocado en el foramen oval con inflado del balón en el cavum de Meckel, comprimiendo el ganglio de Gasser. Anestesia general con intubacion endotraqueal. La punta del catéter se sitúa $1 \mathrm{~cm}$ por detrás del cavum de Meckel y se llena el balón con 0,5-1 $\mathrm{ml}$ de contraste radiológico al 50\% para corroborar la posición correcta (forma de pera). Se genera una presión entre 60 y $1.200 \mathrm{mmHg}$ mantenida durante 0,5-10 min. Este método es de gran utilidad terapéutica y de escaso riesgo, aunque durante la intervención puede darse una bradicardia severa con hipotensión que limita su indicación (17). Deja una 
TABLA V. TECNICAS INTERVENCIONISTAS NT

\begin{tabular}{|c|c|c|c|c|c|c|c|c|c|}
\hline Nivel & Dificultad & $\begin{array}{l}\text { Procedimiento } \\
\text { repetible }\end{array}$ & $\begin{array}{l}\text { Déficit } \\
\text { sensitivo }\end{array}$ & Mortalidad & $\begin{array}{l}\text { Posibilidad } \\
\text { recidiva }\end{array}$ & $\begin{array}{l}\text { Remisión } \\
\text { del dolor a: }\end{array}$ & $\begin{array}{c}\text { Indicación } \\
\text { (Estado } \\
\text { general) }\end{array}$ & $\begin{array}{l}\text { Evidencia } \\
\text { radiológica }\end{array}$ & Antestesia \\
\hline \multicolumn{10}{|l|}{ Periférico } \\
\hline $\begin{array}{l}\text { Inyección alcohol } \\
\text { o glicerol }\end{array}$ & + & Sí & Sí & 0 & +++ & Corto plazo & Precario & No & $\begin{array}{c}\text { Local/ } \\
\text { Sedación }\end{array}$ \\
\hline Crioterapia & + & Sí & Sí & 0 & +++ & Corto plazo & Precario & No & $\begin{array}{l}\text { Local/ } \\
\text { Sedación }\end{array}$ \\
\hline Neurectomía & + & Sí & Sí & 0 & +++ & Corto plazo & Precario & No & $\begin{array}{l}\text { Local/ } \\
\text { Sedación }\end{array}$ \\
\hline RF pulsada & + & Sí & No & 0 & $?$ & $?$ & $\begin{array}{c}\text { Todo tipo } \\
\text { de paciente }\end{array}$ & No & $\begin{array}{c}\text { Local/ } \\
\text { Sedación }\end{array}$ \\
\hline \multicolumn{10}{|l|}{ Ganglio de Gasser } \\
\hline $\begin{array}{l}\text { Termocoagulación } \\
\text { radiofrecuencia }\end{array}$ & ++ & Sí & No & + & ++ & $\begin{array}{l}\text { Medio/largo } \\
\text { plazo }\end{array}$ & $\begin{array}{l}\text { Todo tipo } \\
\text { de paciente }\end{array}$ & No & $\begin{array}{l}\text { Local/ } \\
\text { Sedación }\end{array}$ \\
\hline $\begin{array}{l}\text { Rizotomía percutánea } \\
\text { retrogasseriana con } \\
\text { glicerol }\end{array}$ & ++ & Sí & Sí/No & + & ++ & $\begin{array}{l}\text { Medio } \\
\text { plazo }\end{array}$ & $\begin{array}{l}\text { Todo tipo } \\
\text { de paciente }\end{array}$ & No & $\begin{array}{l}\text { Local/ } \\
\text { Sedación }\end{array}$ \\
\hline $\begin{array}{l}\text { Compresión } \\
\text { percutánea con balón }\end{array}$ & ++ & Sí & Sí/No & + & ++ & $\begin{array}{l}\text { Medio } \\
\text { plazo }\end{array}$ & $\begin{array}{c}\text { Todo tipo } \\
\text { de paciente }\end{array}$ & No & General \\
\hline \multicolumn{10}{|l|}{ Fosa posterior } \\
\hline Rizotomía & +++ & Sí/No & Sí & +++ & + & $\begin{array}{c}\text { Medio/largo } \\
\text { plazo }\end{array}$ & Buen estado & Sí & General \\
\hline DMV & +++ & Sí/No & Sí/No & +++ & + & Largo plazo & Buen estado & Sí & General \\
\hline $\begin{array}{l}\text { + Baja } \\
++ \text { Media } \\
+++ \text { Alta }\end{array}$ & & & & & & & & & \\
\hline
\end{tabular}

ligera pérdida de sensibilidad, puede haber pérdida del reflejo corneal, pero no queratitis y tampoco anestesia dolorosa. La recidiva se sitúa entre un $15 \%$ a los 3 años a un $25 \%$ a los 5 (18). Los resultados son simi-

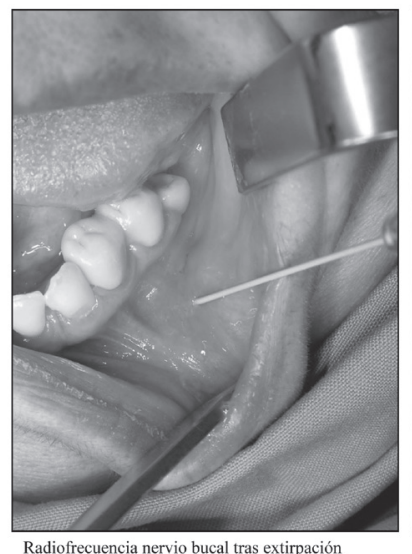

Radiofrecuencia nervio bucal tras extirpación
fibroma yugal

Fig. 2. RF nervio periférico.

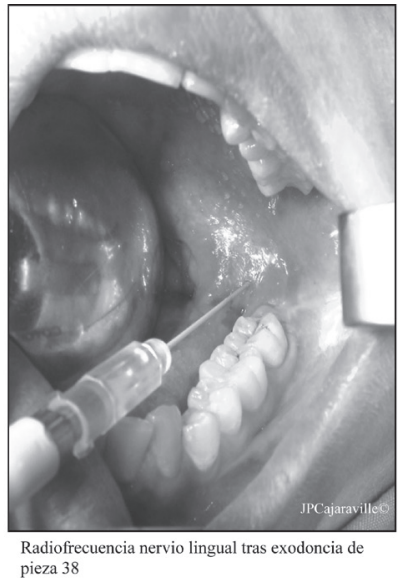
pieza 38 lares a los obtenidos con la termocoagulación pero con mayor índice de complicaciones. Esta técnica se acompaña de alta tasa (60\%) de alteraciones motoras.

- Lesión del ganglio de Gasser por radiofrecuencia: Kirschener, en 1931, sugiere la destrucción del ganglio por procedimientos no quirúrgicos, usando radiofrecuencia (19). Quien perfecciona la técnica es Sweet en 1974, mejorando los resultados y reduciendo las complicaciones del procedimiento (20).

La RF efectuada en el ganglio de Gasser tiene como objetivo acceder al mismo a través del foramen oval, sin necesidad de cirugía abierta (Fig. 3). Actualmente considerado el procedimiento de referencia por su seguridad y eficacia en NT.

- Indicaciones. Entre los síndromes de dolor facial crónico las patologías más frecuentes son la NT y el síndrome de dolor facial atípico. Tanto en el primer caso, ampliamente estudiado, como en el segundo encontramos en la literatura artículos que reflejan su eficacia, aunque en ningún caso aparecen estudios controlados. 


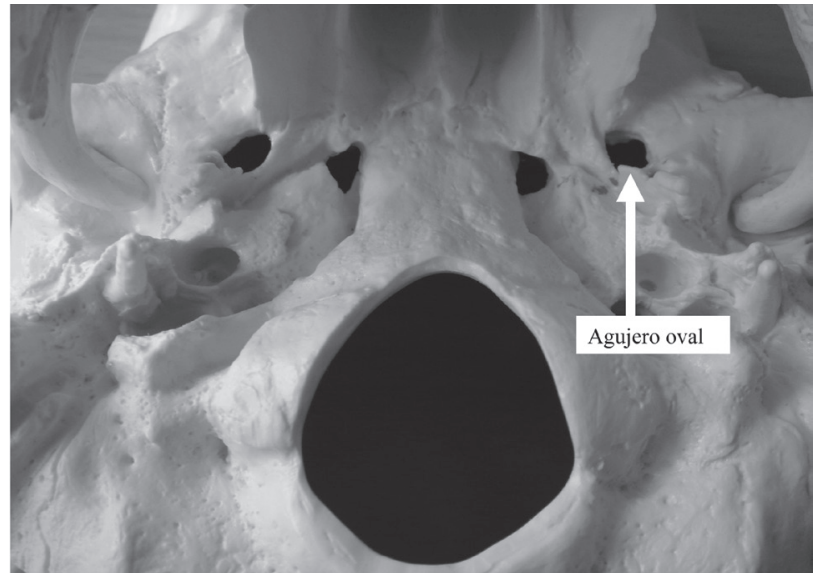

Fig. 3. Foramen ovale.

Las principales indicaciones de la RF sobre el ganglio de Gasser son: NT idiopática, neuralgia secundaria (esclerosis múltiple, anormalidades vasculares y tumores de la fosa posterior), la neuralgia postherpética del trigémino, la cefalea en racimos y la anestesia dolorosa en el área del trigémino, en estos tres últimos supuestos se emplea siempre radiofrecuencia pulsada (RFP) (21). La RF convencional (RFC) no es técnica recomendada en dolor facial atípico (22).

- Técnica; material.

- Generador de RF. Actualmente están comercializados varios sistemas con especificaciones muy similares, siendo característico en alguno de ellos la posibilidad de realizar "radiofrecuencia dual".

- Agujas; $22 \mathrm{G}$ de $10 \mathrm{~cm}$ de longitud, con punta activa de $0,2-0,5 \mathrm{~cm}$. Generalmente se utiliza punta

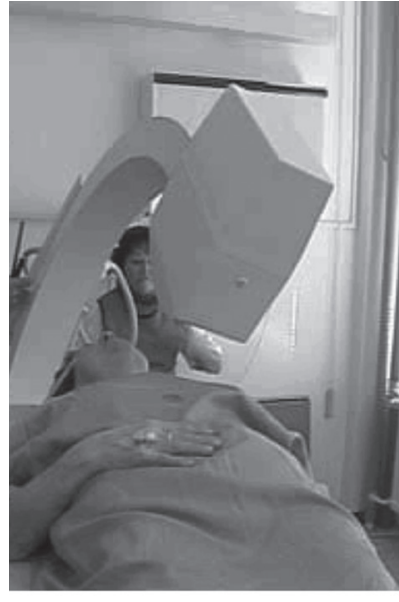

Hiperextensión

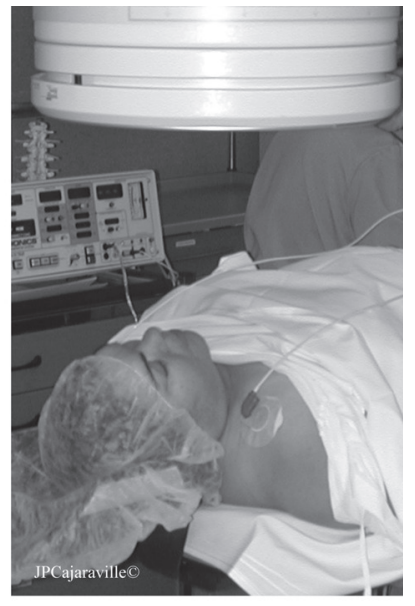

Posición neutra
Fig. 4. Posición del paciente plano tradicional versus posición neutra en fluoroscopia tridimensional.

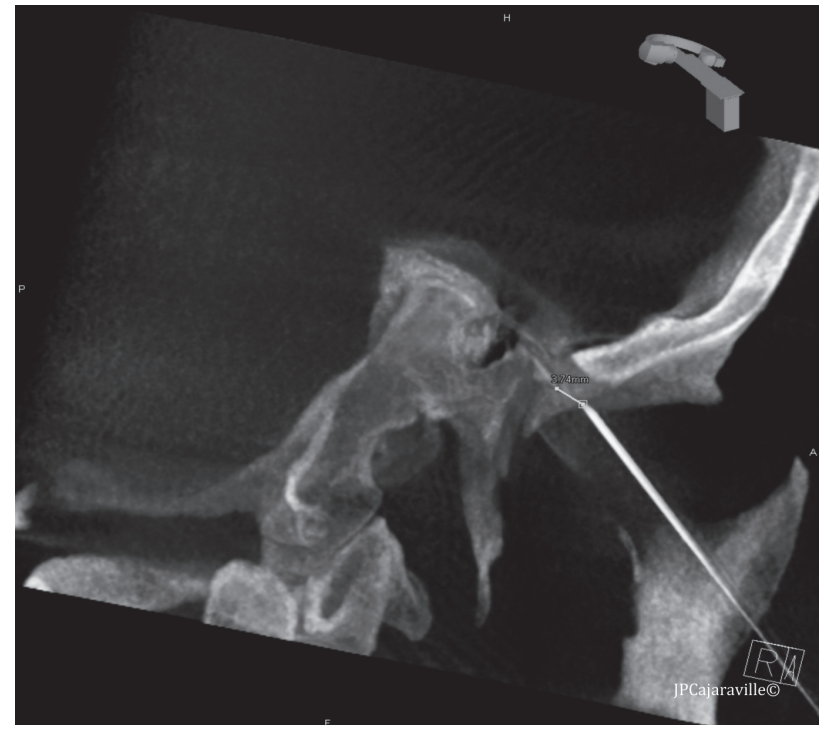

Fig. 5. Imagen representando distancia real $(3,74 \mathrm{~mm})$ de la punta de la aguja a la entrada en el foramen ovale.

activa de $2 \mathrm{~mm}$ para lesiones de una rama y punta activa de $5 \mathrm{~mm}$ para lesiones de varias ramas.

- Posición. El paciente en decúbito supino, fijación cefálica con bandas laterales a la mesa. La hiperextensión $\left(15-20^{\circ}\right)$ de la cabeza, manteniendo el plano fronto-malar paralelo a la mesa, se realiza habitualmente, no obstante, actualmente puede no ser necesario (Fig. 4) con los modernos sistemas de imagen, mediante complejos programas informáticos reconstruyen la base del cráneo en tres dimensiones y/o por la experiencia del especialista en dolor.

- Monitorización. El procedimiento cursa bajo monitorización estándar anestésica y con sedación endovenosa superficial-profunda (propofol y/o remifentanilo). El paciente debe colaborar. Debiendo avisar al anestesista para profundizar en la sedación justo antes de entrar en el agujero oval, pues puede resultar muy doloroso (Fig. 5). Un signo predictivo, aunque no constante, de que se ha atravesado el foramen oval, es la alteración hemodinámica del paciente (hipertensión y bradicardia).

- Referencias anatómicas. Existen dos fases a tener en cuenta durante la realización de la técnica: la penetración a través del foramen oval y la creación de una lesión satisfactoria de las raíces retrogasserianas (23). Si se utilizan sistemas de imagen ordinarios, deberíamos encontrar el punto de punción lateral a la comisura bucal unos 2-3 cm (Fig. 6). Previa infiltración subcutánea con anestésico local se dirige la cánula $3 \mathrm{~cm}$ anterior al conducto auditivo interno y en dirección a la pupila homolate- 

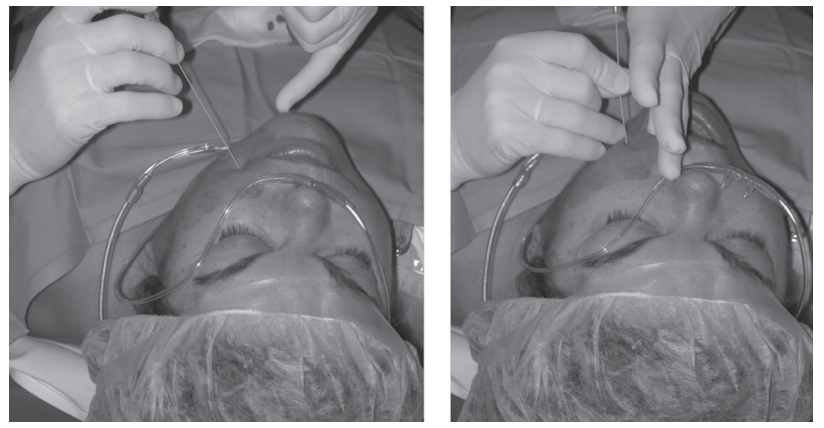

Fig. 6. Lugar de punción de comisura bucal.

ral en posición neutra. Inclinación de la aguja en un plano de 42 a $45^{\circ}$ y su proyección dentaria es hacia el segundo molar para ambos lados. Algunos autores, cada día con menos frecuencia, sugieren la introducción de un dedo en la boca del paciente para asegurar la no punción de la mejilla (Fig. 5). Ayudándonos de fluoroscopia, en visión lateral, avanzamos hasta $2 \mathrm{~mm}$ inferior al clivus.

Todas estas referencias pierden su sentido si manejamos sistemas radiológicos que sean capaces de reconstruir en tiempo real la base del cráneo y visualizar el agujero oval. Con conocimiento exacto de la distancia a la que nos encontramos en todo momento del agujero oval o plano del clivus a la punta activa de la cánula.

El ganglio de Gasser (trigeminal o semilunar), donde se encuentran los núcleos de las neuronas sensitivas, se encuentra localizado en la región petrosa del hueso temporal. Las prolongaciones centrales (postganglionares) forman la raíz del trigémino hasta ingresar al tronco cerebral y mantienen la misma localización espacial con las fibras preganglionares; así, las fibras del nervio oftálmico ocupan la porción anterolateral, la V3 la porción posteromedial y las de la rama maxilar ocupan una topografía intermedia (Fig 7).

- Imagen radiológica. El procedimiento se puede realizar en quirófano o en la sala de angiografía. Clásicamente realizamos una visión oblicua (entre $15-25^{\circ}$ ) y caudal $30^{\circ}$ o más (si fuera posible) hasta visualizar el arco mandibular y foramen ovale (5-10 mm x 3-4 mm). Según estudios anatómicos el agujero oval izquierdo es más grande, con un promedio de perímetro de $3,73 \mathrm{~mm}$ (24). Posición subcigomática oblicua y caudal. El foramen se visualiza medial con relación al arco mandibular y lateral a maxilar (Fig. 7).

Después comprobamos en visión lateral (igualando conductos auditivos en un plano) para localizar silla turca, clivus seno esfenoidal y hueso petroso.

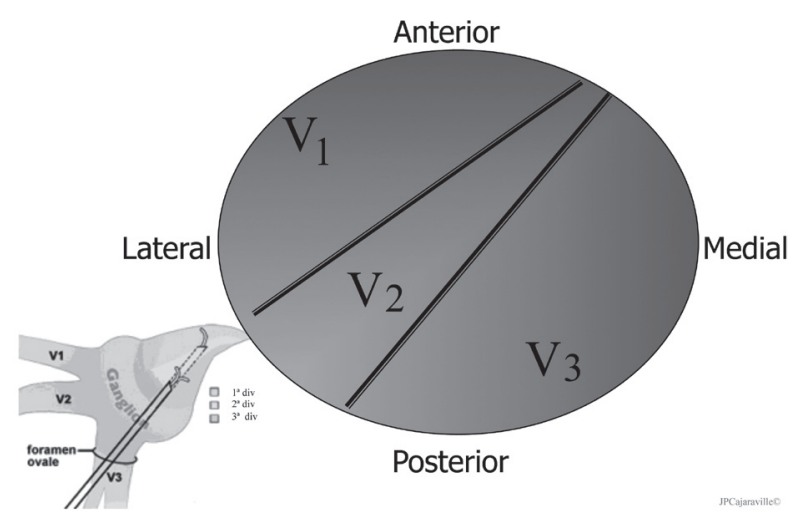

Fig. 7. Ganglio de Gasser.

La punta del electrodo no debe sobrepasar más de $15 \mathrm{~mm}$ al borde anterior del foramen oval (Fig. 9). La imagen y la estimulación son los dos grandes pilares sobre los que se asienta la RF, por ello es primordial ser muy preciso para disminuir tasa de errores y aumentar grado de efectividad de la técnica.

En las figs. 5, 8 y 9 mostramos la diferencia de imagen que obtenemos hoy en día a la hora de realizar nuestra RF con un medio tecnológico actual. Por radiología convencional es muy difícil saber dónde estamos situados dentro del agujero oval, para identificación de la rama utilizaremos la estimulación. Con imágenes que sitúen la punta de la aguja con exactitud, sigue siendo fundamental una buena prueba de estimulación, pues la variabilidad anatómica existe.

- El punto de entrada para $V_{1}$ es más lateral e inferior en el foramen ovale.

- $\mathrm{V}_{3}$ : más medial y superior, medial en el foramen.

- $\mathrm{V}_{2}$ y $\mathrm{V}_{1}$ : el objetivo es al punto medio del agujero oval.

Se debe evitar la canulación de la hendidura esfenoidal en sentido anterosuperior y el foramen yugular en sentido infero posterior.

- Parámetros de estimulación. El electrodo se localiza en la zona retrogasseniana. La respuesta al estímulo es de mayor valor que las marcas radiológicas, de ahí la importancia de tener al paciente consciente durante esta fase.

- Impedancia apropiada: 200-400 W $\Omega$.

- Voltaje: 0-1 voltio.

- Estimulación sensitiva: $50 \mathrm{~Hz}$, anchura de pulso 1 ms. Debe notar parestesia por debajo de 0,5 voltios.

- Estimulación motora: $2 \mathrm{~Hz}$, anchura de pulso 1 ms. No debe apreciarse contracción del masetero a $0,7-1$ voltios.

Si se produce dolor severo durante la estimulación el electrodo podría estar situado sobre el propio 


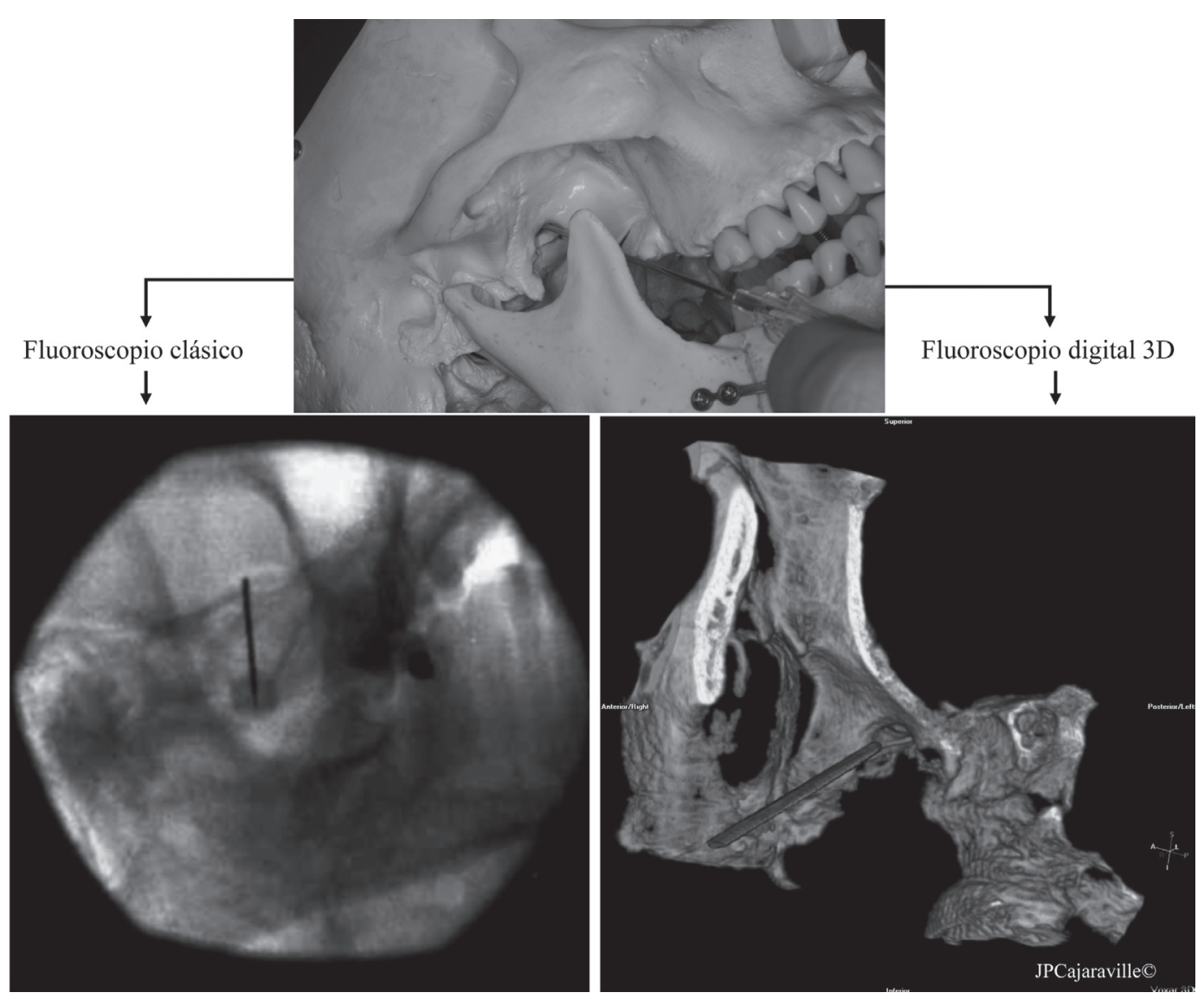

Fig. 8. Abordaje foramen oval.

ganglio o la duramadre (25).

- Parámetros de lesión. La RF convencional (RFC) es el método de elección para NT idiopática, siendo la RF pulsada (RFP) no efectiva (26). La RFP está indicada en dolor facial atípico y NT atípica.

Se realizan inicialmente tres lesiones, aunque podríamos llegar a 4 lesiones en el mismo procedimiento:

- $1 .^{\mathrm{a}}$ : RFC a $65^{\circ} \mathrm{C}$ durante 60 segundos.

- 2. ${ }^{\mathrm{a}}$ : RFC a $70{ }^{\circ} \mathrm{C}$ durante 60 segundos.

- 3. ${ }^{\mathrm{a}} \mathrm{RFC}$ a $72{ }^{\circ} \mathrm{C}$ durante 60 segundos.

Posteriormente a cada lesión debemos comprobar reflejos corneales (Fig. 10) e hipoestesia.

- Efectividad de la técnica. La termocoagulación con radiofrecuencia del ganglio de Gasser es una de las indicaciones de la RF, que aporta mejores resultados clínicos. En un estudio retrospectivo de 25 años se demuestra una mejoría del $97 \%$ que se mantiene cercana al $60 \%$ a los 5 años (27). La tasa de mejoría inmediata está por encima del $95 \%$ en casi todas las series publicadas, con alivio total del dolor cercano al $80 \%$.

Las recidivas son menos frecuentes que con la inyección de glicerol y ligeramente superior a la micro- descompresion vascular. Un $25 \%$ de la población puede recidivar al año. La recurrencia es muy variable y puede llegar al 50\% a los 3 años (Tabla VI).

Otros autores (28) certifican una recurrencia del 20\% a los 7-9 años: un 50-70\% con recurrencia requieren otra intervención quirúrgica y el restante 30-50\% mantienen su dolor bien controlado con medicación.

La tasa de recurrencia varía en función del grado de hipoestesia producido tras la lesión.

- Riesgos. La técnica es repetible, mortalidad prácticamente nula y las complicaciones son poco frecuentes. Aunque la técnica es segura no está exenta de riesgos $(29,30)$, entre las principales complicaciones destacan:

- Hemorragia por punción de la arteria carótida (aguja, demasiado medial e inferior) (0,2-2\%).

- Formación de hematomas retrobulbares.

- Hematoma facial.

- Debilidad del masetero (10-24\%), cuando se realizan lesiones $\mathrm{V}_{3}$. Parestesias que precisan tratamiento $(5-26 \%)$.

- Anestesia dolorosa (0,3-4\%).

- Parálisis de pares craneales (VI, VII, XII) (0,2-6,5\%). 


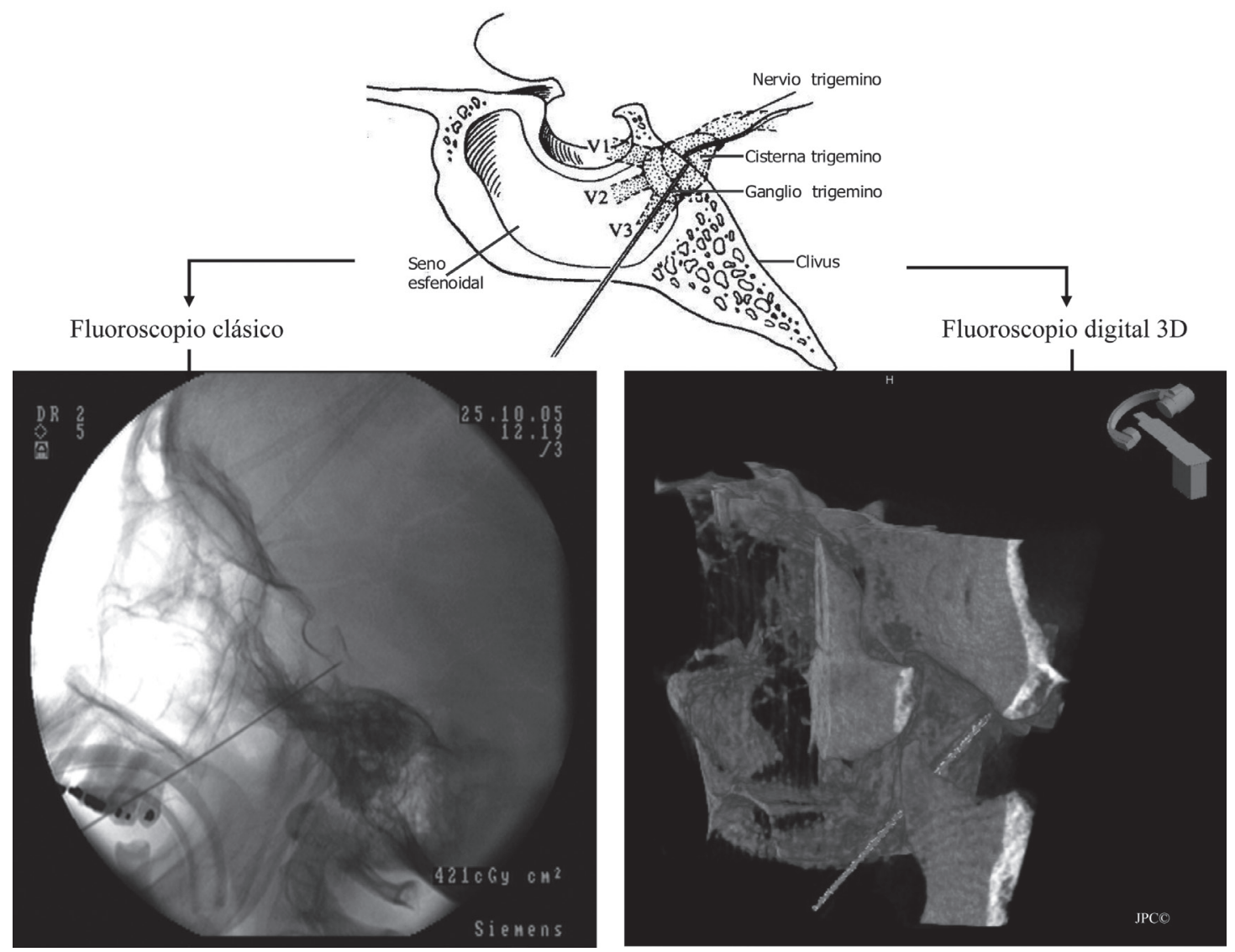

Fig. 9. Visión lateral.

- Parálisis ocular (0,5\%).

- Alteración del reflejo corneal (20\%).

- Queratitis (0,6-1,8\%).

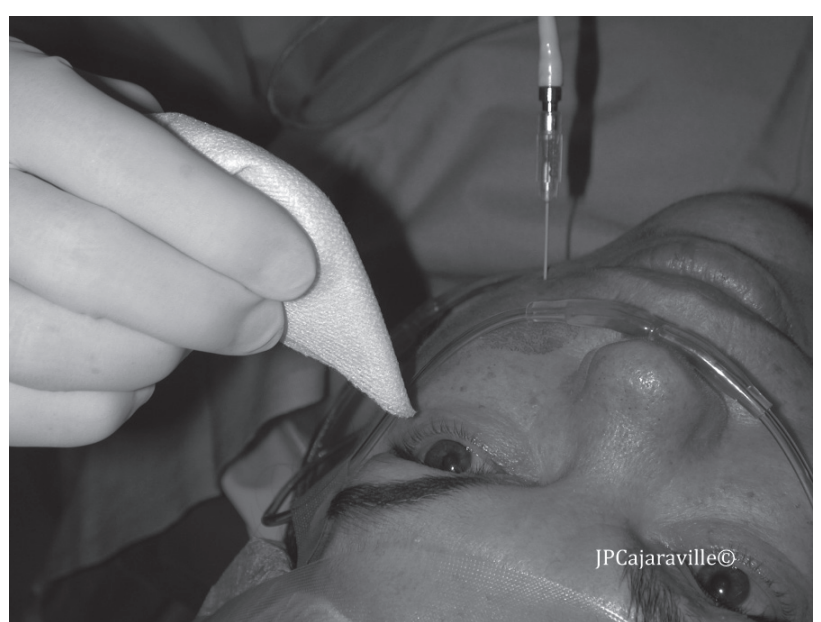

Fig. 10. Comprobación reflejo corneal.
- Rinorrea vasomotora $(0,1 \%)$.

- Dificultad en la audición por tinnitus atribuible a la paresia de pequeños músculos a nivel de la trompa de Eustaquio y membrana timpánica.

Por todo ello, debemos minimizar los riesgos al máximo de nuestras posibilidades, aprovechando los mejores de los recursos que tengamos en nuestro medio hospitalario.

En más del $73 \%$ de los casos se obtiene salida de LCR, y los fracasos en la punción del foramen oval ocurre entre el 0,5-4\% (31). Este último dato, parece lógico pensar, en que se vería disminuido con nuevas técnicas de imagen.

TABLA VI. RECIDIVAS TRAS TRATAMIENTO NT

\begin{tabular}{ll}
\hline Jannetta & 7 años \\
RFC & $2-5$ años \\
Glicerol & 0,5 años \\
Radiocirugía & $2-3$ años \\
\hline
\end{tabular}


Técnicas a nivel de fosa posterior

Los pacientes que presentan una NT secundaria o que no responden al tratamiento médico, deben ser evaluados por un equipo quirúrgico (descompresivas o radiocirugia). La técnica de elección depende de la etiología, edad del paciente, riesgo quirúrgico, etc. La cirugía parece menos efectiva en la NT secundaria a esclerosis múltiple.

\section{CONCLUSIONES}

- Es importante estudiar minuciosamente al paciente con dolor facial, el éxito del tratamiento se fundamenta en el buen diagnóstico.

- Son pocos los estudios metodológicamente correctos para comparar técnicas. En líneas generales la DMV tiene menor tasa de complicaciones siendo la RF una técnica que alcanza cifras más altas y completas de mejoría.

- La radiofrecuencia convencional del trigémino es una sencilla técnica con fácil curva de aprendizaje. La neuroestimulacion es de mayor valor predictivo que marcas radiográficas, de ahí la importancia del paciente consciente durante esta fase, pero creemos que la visualización del agujero oval y la seguridad en la técnica es factor prioritario.

- Procedimientos percutáneos implican un mayor porcentaje de recidiva, aún resulta demasiado pronto para ver si la recurrencia del dolor es significativamente inferior en pacientes visualizados con nuevas técnicas de imagen.

- Se considera a la termocoagulación por radiofrecuencia la primera opción quirúrgica en el paciente anciano con neuralgia trigeminal idiopática.

- Mediante el análisis de decisión coste-efectividad se corrobora a la termocoagulación por radiofrecuencia como la técnica dominante.

\section{Trucos}

1. La técnica se debe realizar en quirófano con sedación y analgesia intravenosa, monitorización continua de la frecuencia cardiaca y la tensión arterial.

2. El lugar de punción en la mejilla se encuentra de 2 a $3 \mathrm{~cm}$ lateral a la comisura de la boca, un poco más lateral e inferior para la primera rama y más medial, para la tercera rama dirige hacia la pupila, no obstante lo importante es visualizar el foramen ovale.

3. Una proyección lateral revela que la aguja se dirige hacia el ángulo producido por el clivus y el reborde petroso.

4. Una vez que se ha penetrado por el agujero oval, se cambia la proyección del rayo para ver la silla turca, el seno esfenoidal y el plano del clivus. Este último será el límite de profundización de la punta de la aguja. A medida que se profundiza se consiguen parestesias en las diferentes ramas, siendo V1 la que se encuentra más profunda.

5. Si la aguja está demasiado medial e inferior, puede penetrarse el canal carotídeo obteniéndose un chorro de sangre arterial pulsátil.

6. El paciente debe ser valorado durante y después de cada lesión. Esto es particularmente importante cuando se realizan lesiones de la primera rama del trigémino.

7. Cuando nos encontramos en una posición adecuada, se obtiene una sensación de hormigueo o de corriente eléctrica en la cara al aplicar un estímulo con una intensidad de 0,1 V. Se utilizan frecuencias de 50 $\mathrm{Hz}$ y una duración de pulso de 1 milisegundo. Si se requiere más de 0,4 a $0,5 \mathrm{~V}$ para obtener respuesta, probablemente necesitaremos reponer el electrodo antes de realizar la lesión.

8. Generar posteriormente lesiones sucesivas a partir de $60{ }^{\circ} \mathrm{C}$ en adelante durante 60 segundos cada lesión, hasta conseguir hipoestesia en la zona deseada de la cara.

9. Cuanto mayor es la hipoestesia generada por la lesión, mayor será el riesgo de anestesia dolorosa.

\section{CORRESPONDENCIA:}

Juan Pérez Cajaraville

Director Unidad del Dolor

Clínica Universidad de Navarra

C/ Pío XII

31008 Pamplona

e-mail: jjperezca@unav.es

\section{BIBLIOGRAFÍA}

1. Pérez-Cajaraville J, Abejón D, Pérez JR. El dolor y su tratamiento a través de la historia. Rev Soc Esp Dolor 2005; 12:373-84.

2. Rose C. Johnson F. Carbamazepina in the treatment of nonseizure disorders: trigeminal neuralgia, other painfun disorders, and affective disorders. Rev Contemp Pharmacother 1997;8:123-43.

3. Tew JM Jr. Treatment of trigeminal neuralgia by percutaneous rhizotomy. Youmans. Vol 6. Second. edition. 1982. p. 3564-79.

4. Szapiro J Jr, Sindou M. Traitement microchirurgical de la neuralgie du trijumeau: resultats et facteurs pronostiques de la decompression vasculaire microchirurgicale. Neurochirurgie, 1986;32:103-21.

5. Wall P. Melzack R. Textbook of Pain. 1er. edition.Churchil Livington 1994.

6. Goetz CG. Textbook of Clinical Neurology. 2nd ed. St. Louis, Mo: WB Saunders; 2003. p. 178-9.

7. Zakrzewska JM. Trigeminal neuralgia. Londres: W.B. Saunders Co. Ltd., 1995. 
8. Attal N. New advances in the treatment of neuropathic pain. Acta Neurologica Scandinavica. 1999;100(173) Supplement.

9. Adamczyk M, Bulski T, Sowiska J, et al. Trigeminal nerveartery contact in people without trigeminal neuralgia-MR study. Med Sci Monit. 2007 May;133 suppl 1:38-43.

10. Fromm G, Terrence C, Maroon J. Trigeminal neuralgia. Current concepts regarding etiology and pathogenesis. Arch Neurol 1984;41:1204-7.

11. Hunt K, Patwardhan R. Trigeminal neuralgia: a modern-day review. Int Rev Neurobiol. 2007;79:621-31. PMID: 17531861

12. Perez-Cajaraville J, Honorato J. Plan maestro en abordaje integral del dolor. Modulo 3 . 2004.

13. Bajwa ZH, et al. Trigeminal neuralgia. Waltham (MA): UpToDate; 2006.

14. McLeod NM, Patton DW. Peripheral alcohol injections in the management of trigeminal neuralgia. Oral Surg Oral Med Oral Pathol Oral Radiol Endod. 2007 Jul;104(1):12-7. Epub 2007 Apr 11.

15. Fardy MJ, Patton DW. Complications associated with peripheral alcohol injections in the management of trigeminal neuralgia. Br J Oral Maxillofac Surg 1994;32:387-91.

16. Bittar GT, Graff-Radford SB. The effects of streptomycin/ lidocaine block on trigeminal neuralgia: a double blind crossover placebo controlled study. Headache 1993;33:155-60.

17. Skirving DJ, Dan NG. A 20-year review of percutaneous balloon compression of the trigeminal ganglion. J Neurosurg 2001;94(6):913-7.

18. Brown JA, McDaniel MD, Weaver MT. Percutaneous trigeminal nerve compression for treatment of trigeminal neuralgia: results in 50 patients. Neurosurgery 1993;32:570-3.

19. Kirschner M. Die Behandlung der Trigeminusneuralgia. Munchen Med Wschr 1942;89:235-9.

20. Sweet W, Wepsic J. Controlled thermocoagulation of the trigeminal ganglion and rootlets for differential destruction of pain fibres. Trigeminal neuralgia. J Neurosurg $1974 \mathrm{~b}$; 39:143-56.
21. Ruiz-López R, Erdine S. Treatment of cranio-facial pain with radiofrequency procedures. Pain Practice 2002;2(3):206-13.

22. Teixeira MJ, Siqueira SR. Percutaneous radiofrequency rhizotomy and neurovascular decompresion of the trigeminal nerve for the treatment of facial pain. Arq Neuropsiquiatr. 2006 Dec;64(4):983-9.

23. López-Rodríguez MA, Varela M, Camba MA. Uses of radiofrequency for the management of benign $\mathrm{c} h$ ro nic pain. A review of the literature published in the past 6 years. Rev Soc Esp Dolor 2001;8:397-411.

24. García HMG, Sánchez Rodríguez JP, Tenopala Villegas S. Neuralgia del trigémino An Med (Mex) 2012;57(1):39-47.

25. Alonso Galán JC, Reyes Téllez Girón J, Tomasini Ortíz P, et al. Craneometría del agujero oval aplicada al tratamiento de la neuralgia trigeminal Arch Neurocien (Mex) 2010; 15(3):152-5.

26. Erdine S, Ozyalcin NS, Cimen A, et al. Comparison of pulsed radiofrequency with conventional radiofrequency in the treatment of idiopathic trigeminal neuralgia. Eur. J Pain, 2007 Apr;11(3):309-13.

27. Kanpolat Y, Savas A, Bekar A, Berk C. Percutaneous controlled radiofrequency trigeminal rhizotomy for the treatment of idiopatic trigeminal neuralgia: 25 years experience with 1600 patients. Neurosurgery 2001;48:524-34.

28. López-Rodríguez MA, Varela M, Camba MA. Aplicaciones de la radiofrecuencia en el tratamiento del dolor crónico benigno una revisión de las publicaciones de los últimos 6 años. Red Soc. Esp. Dolor, 2001;8:397-411.

29. Taha JM, Tew JM. Comparison of surgery treatments for trigeminal neuralgia: reevaluation of radiofrequency rhizotomy. Neurosurgery 1966;38:865-71.

30. Racz GB, Erdine S: Trigeminal ganglion procedures. In: Raj PP (editor). Textbook of Regional Anesthesia. Churchill Livingstone 2002. p. 801-8.

31. Gybels J, Sweet W. The trigeminal nerve. In: Gildenberg (editor). Neurosurgical treatment of persistent pain. Basel: Karger; 1989. p. 1-69. 